\title{
CALIBRATION OF THE REFLECTED SOLAR INSTRUMENT FOR THE CLIMATE ABSOLUTE RADIANCE AND REFRACTIVITY OBSERVATORY
}

\author{
Kurtis Thome ${ }^{1}$, Robert Barnes ${ }^{2}$, Rosemary Baize ${ }^{3}$, Joseph O’Connell ${ }^{3}$, Jason Hair ${ }^{1}$ \\ ${ }^{1}$ NASA, Goddard Space Flight Center; ${ }^{2}$ Science Applications Int’l. Corp.; ${ }^{3}$ NASA Langley Research \\ Center
}

\begin{abstract}
The Climate Absolute Radiance and Refractivity Observatory (CLARREO) plans to observe climate change trends over decadal time scales to determine the accuracy of climate projections. The project relies on spaceborne earth observations of SI-traceable variables sensitive to key decadal change parameters. The mission includes a reflected solar instrument retrieving at-sensor reflectance over the 320 to $2300 \mathrm{~nm}$ spectral range with $500-\mathrm{m}$ spatial resolution and $100-\mathrm{km}$ swath. Reflectance is obtained from the ratio of measurements of the earth's surface to those while viewing the sun relying on a calibration approach that retrieves reflectance with uncertainties less than $0.3 \%$. The calibration is predicated on heritage hardware, reduction of sensor complexity, adherence to detector-based calibration standards, and an ability to simulate in the laboratory onorbit sources in both size and brightness to provide the basis of a transfer to orbit of the laboratory calibration including a link to absolute solar irradiance measurements.
\end{abstract}

Index Terms - Global climate change, CLARREO, SItraceability, radiometric calibration

\section{INTRODUCTION}

The Climate Absolute Radiance and Refractivity Observatory (CLARREO) mission addresses the need to observe high-accuracy, long-term climate change trends and to use decadal change observations as the most critical method to determine the accuracy of climate change projections such as those in the IPCC Report[1]. A rigorously known accuracy of both decadal change observations as well as climate projections is critical in order to enable sound policy decisions. The CLARREO Project will implement a spaceborne earth observation mission designed to provide rigorous SI traceable observations (i.e., radiance, reflectance, and refractivity) that are sensitive to a wide range of key decadal change variables, including:

1) Surface temperature and atmospheric temperature profile

2) Atmospheric water vapor profile
3) Far infrared water vapor greenhouse

4) Aerosol properties and anthropogenic aerosol direct radiative forcing

5) Total and spectral solar irradiance

6) Broadband reflected and emitted radiative fluxes

7) Cloud properties

8) Surface albedo

There are two methods the CLARREO mission will rely on to achieve these critical decadal change benchmarks: direct [2] and reference inter-calibration. A quantitative analysis of the strengths and weaknesses of the two methods has led to the recommended CLARREO mission approach. The project consists of two satellites launched into 90degree, precessing orbits separated by 90 degrees. The instrument suite receiver on each spacecraft includes one emitted infrared spectrometer, two reflected solar spectrometers: dividing the spectrum from ultraviolet through near infrared, and one global navigation receiver for radio occultation. The measurements will be acquired for a period of three years minimum, with a five-year lifetime goal, enabling follow-on missions to extend the climate record over the decades needed to understand climate change.

The current work concentrates on the reflected solar instrument giving an overview of its design and calibration approach. The calibration description includes the approach to achieving an SI-traceable system on orbit. The calibration overview is followed by a preliminary error budget based on techniques currently in place at the National Institute of Standards and Technology (NIST).

\section{REFLECTED SOLAR INSTRUMENT DESCRIPTION}

The basis of the design of the RS sensor is the retrieval of an at-sensor reflectance over the spectral range from 320 to $2300 \mathrm{~nm}$ with $500-\mathrm{m}$ GIFOV and a $100-\mathrm{km}$ swath width. Reflectance is obtained from the ratio of measurements of radiance while viewing the earth's surface to measurements of irradiance while viewing the sun. The need to measure the energy leaving the earth's surface as well as the solar 
irradiance means that signals vary by factors of 2 to 10 due to multi-dimensionality of the problem caused by:

- $\quad$ Surface reflectance changes

- View/solar geometry (seasonal and geographic)

- $\quad$ Atmospheric effects

- Spectral variation

The RS instrument must be designed to account for these effects as well as include a calibration approach that allows accurate retrieval of the reflectance traceable to SI standards at a level better than $0.3 \%$ in the mid-visible. Such a required accuracy provides a data set that, when collected globally, reduces sampling biases for climatologically significant spatial and temporal averages over annual means [3].

The calibration approach taken in order to achieve the ambitious $0.3 \%$ absolute calibration uncertainty is predicated on a reliance on heritage hardware, reduction of sensor complexity, and adherence to detector-based calibration standards. One design being evaluated currently for the reflected solar instrument is based on an Offner spectrometer which is capable of limiting spectral smile on the focal plane. The design relies on two separate focal planes each with its own entrance aperture and grating. The two separate focal planes cover spectral ranges of 320-640, $600--2300 \mathrm{~nm}$ implemented as two individual spectrometers.

\section{CALIBRATION OVERVIEW}

The system design currently being evaluated relies on a direct solar view as the primary calibration approach while on orbit. The data from a solar view are coupled with the earth view data and knowledge of the sensor optical geometry to retrieve at-sensor reflectance. The method is similar in concept to past sensors that rely on solar diffuser data to derive reflectance. One reason for adopting a reflectance philosophy is it reduces the need for elaborate onboard calibration sources. Conversion of the reflectance to an absolute radiance will rely on access to an appropriately accurate solar irradiance.

Reliance on a direct solar view requires that the sensor reduces the incident solar energy to a level comparable to the earth-viewing energy, approximately a factor of 50,000. The approaches being evaluated are a single pinhole aperture, neutral density filters, a collection of pinhole apertures, and combinations of these three. The attenuators require extremely careful evaluation during ground testing, but are also a source of uncertainty on orbit if the attenuators degrade in some fashion. Evaluation of the attenuators on orbit takes place through coordinated views of the sun and the moon. The brightness of the moon is low enough to permit measurements without the attenuators allowing the coupled lunar/solar views to determine if the attenuators are operating properly.

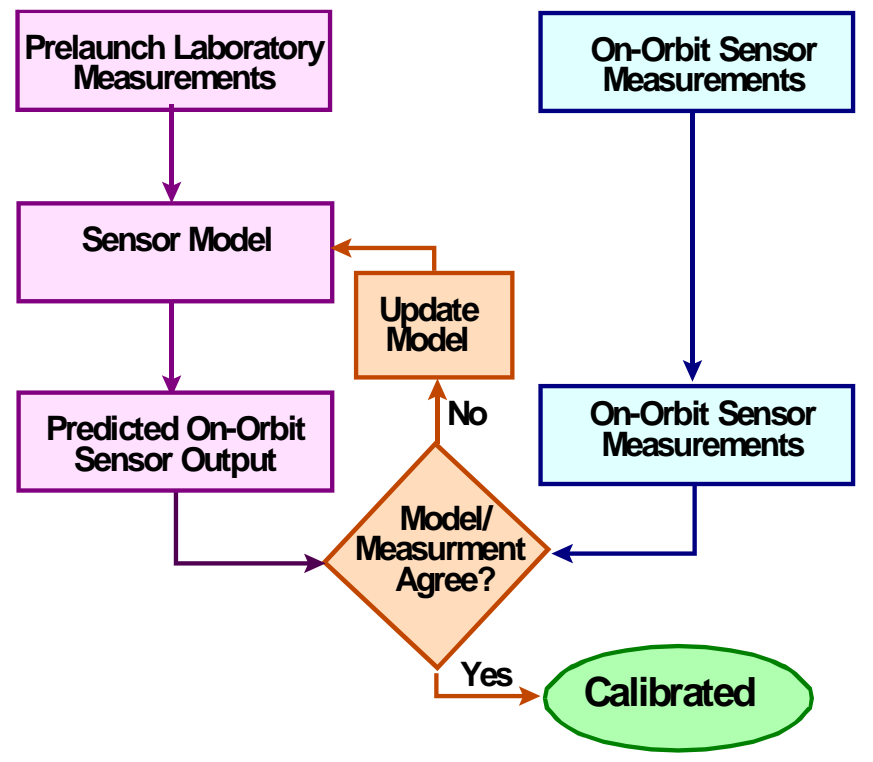

Figure 1. Flow chart demonstrating the calibration process for the CLARREO RS instrument.

The reason that three attenuator approaches are currently under study is that an additional goal of CLARREO calibration is to rely on multiple and independent calibration approaches. Thus, other on-orbit validation approaches such as aircraft underflights and ground validation sites are planned. Multiple calibration pathways during preflight characterization including solar-like sources and a hyperspectral image projector will be used.

A critical part of the calibration process is the development of an SI-traceable data set. The SI-traceability is achieved by characterizing the sensor to SI-traceable, absolute radiometric quantities during prelaunch calibration, primarily the electric Watt. This effort is illustrated as the prelaunch calibration box shown in Figure 1. Also included in that box is the absolute calibration of the sensor in irradiance mode and radiance mode. and the determination of geometric factors for conversion to reflectance.

The end result of the prelaunch calibration is sufficient data to develop a full sensor model that can predict expected sources to be used for on-orbit calibration: sun, moon, and other planetary/stellar sources. Such an approach permits the on-orbit calibration to "validate" the prelaunch calibration. Agreement between prelaunch and on-orbit (as shown in Figure 1) means the system is calibrated, and by analogy traceable to the pre-launch SI measurements. Disagreement is inferred to mean an that the sensor model requires improvement based on the on-orbit data, and would likely include an additional set of characterization measurements. Once the on-orbit traceability is established with a demonstrable error budget, the solar and lunar views provide information regarding temporal changes in the sensor. 


\section{ERROR BUDGET ANALYSIS}

Clearly, the key is to ensure the prelaunch calibration simulates on-orbit sources at sufficient accuracy to meet the CLARREO requirements. This includes absolute irradiance calibration for solar view and simulated geometry of solar and lunar views for stray light. The reason stray light plays such a large role in the prelaunch calibration is that a preliminary error budget analyses led to uncertainty estimates in excess of $1.5 \%$ in the mid-visible. The dominant error source identified in that original analysis was how stray light behaves differently between the solar and the terrestrial views. The use of a two-aperture, two-grating design reduces stray light but does not eliminate it. Thus a measurement scheme is needed that has well understood error budgets and can provide the sensor information needed for a CLARREO RS instrument model.

Calibration systems, such as NIST's Spectral Irradiance and Radiance Responsivity Calibrations using Uniform Sources (SIRCUS) facility, provide such standards and a capability to understand stray light, spectral response, and polarization sensitivity at the level necessary for CLARREO [4]. The basis of SIRCUS is a well-understood tunable laser source that can be coupled to a fiber optic system providing both radiance and irradiance sources. The output of the source is determined via detector standards characterized against the Primary Optical Watt Radiometer (POWR).

Figure 2 illustrates the traceability of a SIRCUS system with a POWR laser source being calibrated against a cryogenic substitution radiometer. That laser source is used to calibrate the absolute response of what is termed the

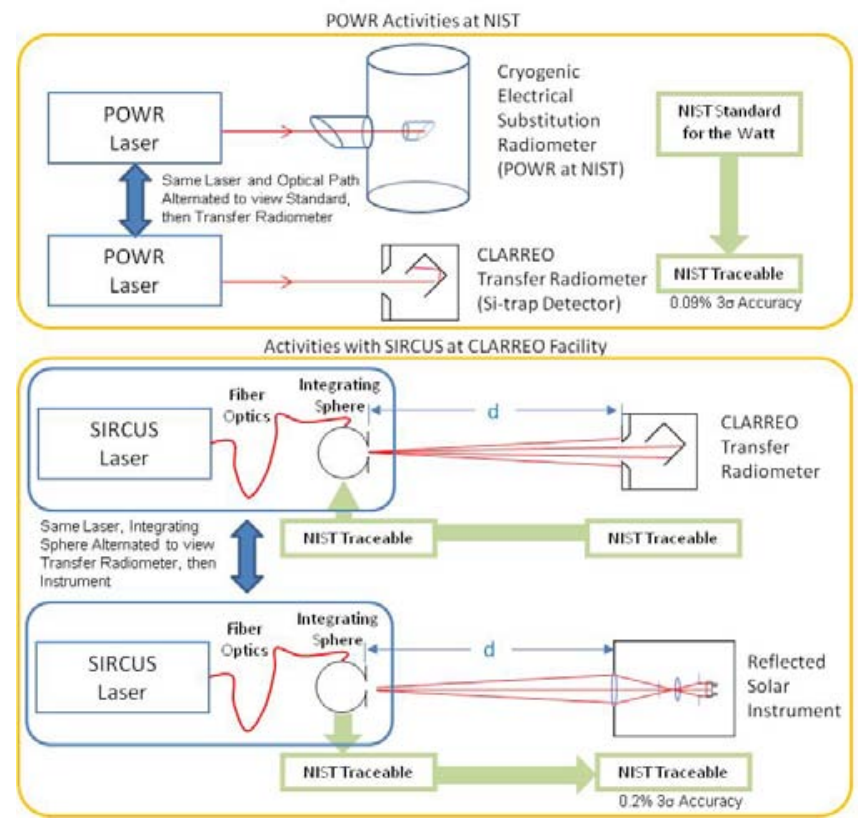

Figure 2. Diagram illustrating the traceability of a SIRCUS-like approach for a radiance calibration of CLARREO RS. the CLARREO transfer radiometer. The transfer radiometer determines the absolute output of the tunable source which in turn provides the source needed for the calibration of the CLARREO RS instrument.

Work by NIST with their laboratory version of SIRCUS has shown that stray light sensitivity of an imaging system can be determined to an order of magnitude better than methods widely used in other radiometric calibration facilities. While the NIST laboratory SIRCUS is currently not a viable system for the flight version of the CLARREO RS sensor, studies currently underway are evaluating the best approach for making a SIRCUS-like system available for CLARREO.

Inclusion of a detector-based standard such as those in SIRCUS provides the capability for absolute radiometric calibration with uncertainties $<0.2 \%$ for a radiance source. This calibration is traceable to the electrical Watt (the SI quantity). Figure 3 shows the dominant expected error sources in retrieving the at-sensor reflectance. The earth:solar ratio term is that uncertainty from the ratio of the earth and solar view data. Error sources in the ratio are from uncertainties in the earth measurement, the solar measurement, and of the relative spectral response.

Details of the earth view error are shown in Figure 4. The values given in the figure are based on current state of the art approaches and a well-behaved sensor. These values have yet to be achieved for current terrestrial imaging sensors, primarily due to stray light uncertainties leading to errors that have not warranted the efforts needed to reduce the other error sources. Confidence in achieving the errors listed is based on work with non-imaging sensors.

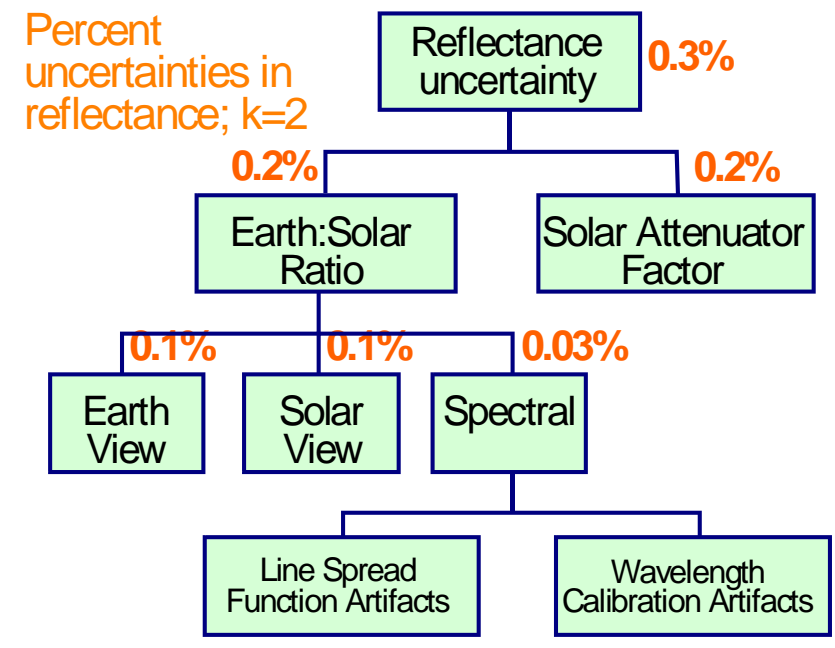

Figure 3. Preliminary error budget and key error sources for the CLARREO RS instrument. 


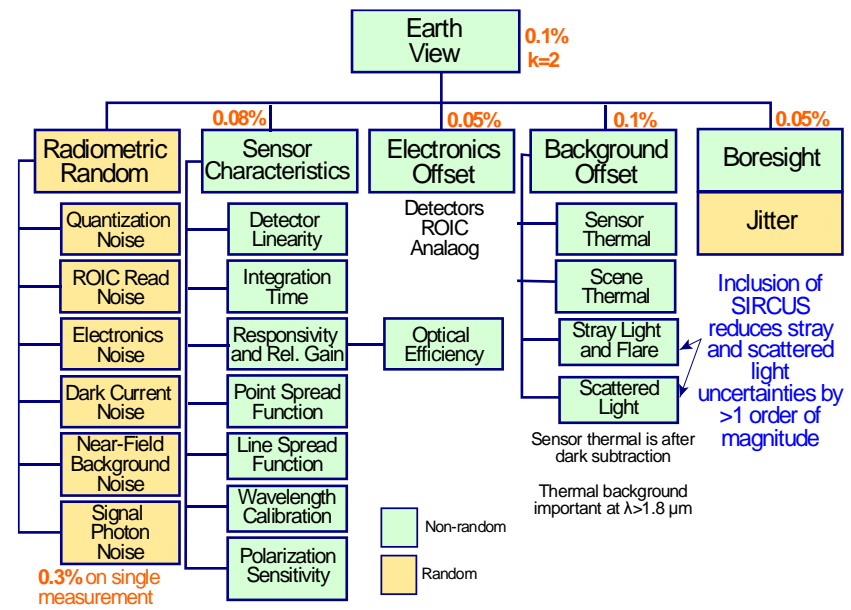

Figure 4. Error sources expected for the earth view, including random uncertainties on the lefthand column and systematic uncertainties in other columns.

The solar view uncertainty in listed in Figure 3 has a similar set of error sources as those shown in Figure 4 for the earth view but also includes terms related to the behavior of the solar attenuator during the solar view. Uncertainty in the solar attenuator characterization is shown in Figure 3

Obviously, the values shown in Figures 3 and 4 require demonstration, and this is especially true in the shortwave infrared where the transfer radiometer detector standards are not well understood at the current time. Efforts to demonstrate these error budgets will take place during breadboard developments of the sensor and will include close collaboration with NIST.

The SIRCUS-like source provides the opportunity to evaluate the RS system with sources that can simulate the brightness and spatial shape of expected on-orbit calibration sources. Unfortunately, the SIRCUS-like system provides a near-monochromatic source while the on-orbit sources (sun, earth, moon) are broadband sources. A further issue is that sources that can provide the broadband spectral and the spatial nature of the on-orbit sources currently have larger uncertainties than the CLARREO requirements[5]. A combination of the SIRCUS narrow-band sources and the broadband laboratory sources will provide CLARREO with the needed accuracy as well as the information needed to develop the instrument model.

\section{CONCLUSIONS}

The goals of CLARREO including the reflected solar instrument are daunting but achievable. Characterization of the reflected solar instrument relies on improvements to currently-available calibration approaches. Careful sensor design to limit stray light and polarization sensitivity also is critical to achieving the needed accuracy. In the end, adherence to these basic design and characterization principles will provide data at the accuracy required to provide the basis for a set of data records needed to understand the earth's climate.

\section{REFERENCES}

[1] Intergovernmental Panel on Climate Change. Climate change 2007: the physical science basis. Contribution of Working Group I to the Fourth Assessment Report of the Intergovernmental Panel on Climate Change. Geneva, Switzerland, 2007.

[2] S. Leroy, J. Anderson, J. Dykema, and R. Goody, "Testing climate models using thermal infrared spectra." Journal of Climate, pp. 1863, 2008.

[3] B. J. Soden, I. M. Held, R. Colman, K. M. Shell, J. T. Kiehl, and C. A. Shields, "Quantifying Climate Feedbacks Using Radiative Kernels." Journal of Climate, Vol. 21, pp. 3504-3520, 2008.

[4] Brown, S.W., Eppeldauer, G.P., and Lykke, K.R., "NIST facility for Spectral Irradiance and Radiance Responsivity Calibrations with Uniform Sources,” Metrologia Vol. 37, 579-582, 2000.

[5] Rice, J. P., Brown, S. W., Johnson, B. C., and Neira, J. E., "Hyperspectral image projectors for radiometric applications," Metrologia Vol. 43, S61-S65, 2006. 